\title{
Multi-Objective Optimization of the Dressing Parameters in Fine Cylindrical Grinding
}

\author{
Irina Stefanova Aleksandrova \\ Technical University of Gabrovo, Bulgaria
}

The optimum conditions for dressing grinding wheels determined and recommended in the literature are valid only for particular types and tools of dressing and grinding. In this paper, an attempt has been made to optimize the dressing process parameters in fine cylindrical grinding. To define the optimum values of the dressing process variables (radial feed rate of diamond roller dresser $f_{r d}$, dressing speed ratio $q_{d}$, dress-out time $t_{d}$, diamond roller dresser grit size/grinding wheel grit size ratio $q_{g}$, type of synthetic diamonds and direction of dressing), a multi-objective optimization has been performed based on a genetic algorithm. In the capacity of the optimization parameter, a generalized geometric-mean utility function has been chosen, which appears to be a complex indicator characterizing the roughness and accuracy of the ground surface, the grinding wheel lifetime and the manufacturing net costs of the grinding operation. The optimization problem has been solved in the following sequence: 1) a model of the generalized utility function has been created reflecting the complex effect of the dressing system parameters; 2) the optimum conditions of uni-directional and counter-directional dressing of aluminium oxide grinding wheels by experimental diamond roller dressers of synthetic diamonds of AC32 and AC80 types and different grit size at which the generalized utility function has a maximum have been determined; 3) a Pareto optimum solution has been found $\left(f_{r d}=0.2 \mathrm{~mm} / \mathrm{min} ; q_{d}=0.8 ; t_{d}=4.65 \mathrm{~s}\right.$; $\left.q_{g}=2.56\right)$, which guarantees the best combination between the roughness and the deviation from cylindricity of the ground surface, the grinding wheel lifetime and the manufacturing net costs of the grinding operation.

Keywords: fine cylindrical grinding, dressing parameters, diamond roller dressers, multi-objective optimization

Highlights

- A new multi-objective optimization approach based on a genetic algorithm and a generalized utility function to define the optimum values of the dressing system parameters in fine cylindrical grinding has been performed.

- $\quad$ Regression models for the response variables of the fine grinding process depending on the dressing system parameters have been built.

- Theoretical-experimental models have been created for determining the generalized utility function as a complex indicator characterizing the response variables of the fine grinding process.

- The optimum conditions of the uni-directional and counter-directional dressing of aluminium oxide grinding wheels by diamond roller dressers of synthetic diamonds of AC32 and AC80 types have been determined.

- A Pareto optimum solution has been found that guarantees the best combination between the roughness and the deviation from the cylindricity of the ground surface, the grinding wheel lifetime, and the manufacturing net costs.

\section{O INTRODUCTION}

The grinding process is characterized by a great number of response variables: economic (production rate, net costs), dynamic (cutting forces and power rate), and manufacturing (grinding wheel lifetime and cutting ability, roughness and accuracy of the machined surface). It has been found that these variables depend both on the cutting conditions during grinding and on the micro- and macro-geometry of the grinding wheel cutting surface formed during dressing [1] to [4].

The dressing process and its effect on the grinding response variables were studied in several publications. Cebalq [5] found that the different combinations between the dressing mode, dressing conditions, and the grinding wheel specification lead to different inter-grit spacing between the abrasive grits of the grinding-wheel cutting surface and, as a result, different response variables of the grinding process (equivalent grinding thickness, specific metal removal rate, roughness of the grinding-wheel cutting surface, roughness of the ground surface, etc.). Baseri et al. [6] and [7] and Baseri [8], Wegener et al. [9] and Palmer et al. [10] proved that the grindingwheel topography and the conditions under which it is prepared have a profound influence upon the grinding performance, defined by the grinding forces, the power consumption, the cutting zone temperature, the radial wear of the wheel and also the surface finish of the workpiece. Chen et al. [11] found that a satisfied and stable grinding process can be controlled in real-time by means of utilizing the combination of optimal parameters, such as spindle speed, effective pack density, and the cutting space of abrasive grits. A similar conclusion is also drawn in the publications of other authors [12] to [15]. 
Despite the significant influence of the dressing process on the response variables of the grinding process, its setup is often done based on the experience of the qualified staff or with the help of data handbooks [5], [8] and [16]. However, the dressing conditions selected by such practice are usually standard and they cannot satisfy certain economic criteria. Therefore, recently, some researchers [17] to [22] have applied, during cylindrical grinding, various techniques to optimize the grinding process parameters (grinding wheel speed, workpiece speed, depth of dressing, lead of dressing, contact area, grinding fluid, etc.) using a multi-objective function model with a weighted approach. The production costs, the production rate and the surface quality have been evaluated for the optimal grinding conditions, subject to constraints of thermal damage, wheel-wear parameters, and machine-tool stiffness. Amitay et al. [23] describes a technique for grinding and dressing optimization so that the maximum workpiece removal rate is ensured subject to constraints on workpiece burn and surface finish in an adaptive control grinding system. In his study, Baseri [8] used a feed-forward backpropagation neural network and a simulated annealing algorithm for the simultaneous minimization of the tangential cutting force and the surface roughness. During the experimental procedure, the grinding conditions were constant while the dressing conditions varied. The dressing parameters considered during the experiments were the dressing speed ratio, the dressing depth, and the dressing cross-feed. Klancnik et al. [24] presented a new and effective method of multi-criterion optimisation based on the evolutionary approach. This method can be introduced into the process of automatic programming of machine tools, including grinding machines. The analysis of the results provided by some authors in [8] and [17] to [22] shows that the optimization of the grinding process, depending on the dressing conditions, is a complicated non-linear optimization problem with constraints and multi-modal characteristics. The optimization problems have been solved under specific conditions of grinding and dressing. The defined optimum dressing parameters depend on the dressing method and dressing tool used. Further difficulties during optimization are associated with the fact that no comprehensive grinding models exist relating the dressing conditions to all response variables of the grinding process. At present, there is no comprehensive mathematical model that encompasses all aspects of grinding. In addition, the objective of optimization can vary depending on its application. All this shows that the optimization of the dressing conditions during cylindrical grinding should be performed considering the particular grinding and dressing conditions.

In a previous study [25], the results of multiobjective optimization of dressing grinding wheels made of aluminium oxide by using diamond roller dressers with medium- and high-strength synthetic diamonds of $\mathrm{AC} 32$ and $\mathrm{AC} 80$ types with different grit size during rough cylindrical grinding were presented. The generalized utility function was chosen in the capacity of the optimization parameter. The defined optimum dressing system parameters (dressing speed ratio, radial feed rate of the diamond roller dresser, dress-out time, diamond roller dresser grit size/grinding wheel grit size ratio, type of synthetic diamonds and direction of dressing) guarantee the maximum lifetime and maximum cutting ability coefficient of grinding wheels, the minimum tangential cutting force, and the maximum production rate of the grinding process. Taking into account the fact that grinding is widely used as a finishing process, it is necessary to define the dressing system parameters providing minimum roughness and maximum accuracy of the ground surfaces, together with maximum lifetime of the grinding wheel and minimum manufacturing net costs.

The objective of this paper is to determine the optimum dressing system parameters for grinding wheels made of aluminium oxide with experimental diamond roller dressers of medium- and high-strength synthetic diamonds of $\mathrm{AC} 32$ and $\mathrm{AC} 80$ types with different grit size during fine cylindrical grinding.

\section{STUDY AND MODELLING OF FINE GRINDING RESPONSE VARIABLES}

\subsection{Equipment, Materials, and Methods}

The task of this study is to find the correlations between the fine cylindrical grinding response variables and the parameters of uni-directional and counter-directional dressing of grinding wheels by employing diamond roller dressers, which have a layer of medium- and high-strength synthetic diamonds. The dressing speed ratio $q_{d}$, the radial feed rate $f_{r d}$ $[\mathrm{mm} / \mathrm{min}]$, the dress-out time $t_{d}[\mathrm{~s}]$ and the ratio $q_{g}$ between the grit sizes of the diamond roller dresser and grinding wheel are selected as control factors.

The experimental studies have been carried out on a KUF 250/500 cylindrical grinder (Fig. 1) under the following grinding conditions: grinding wheels: $1-350 \times 125 \times 22.5, \quad 95 \mathrm{~A} 80 \mathrm{~K} 8 \mathrm{~V} 38, \quad 95 \mathrm{~A} 60 \mathrm{~K} 8 \mathrm{~V} 38$, 95A46K8V38, produced by the Abrasive Tools 
Table 1. Diamond grit properties [27] to [29]

\begin{tabular}{|c|c|c|c|c|}
\hline \multirow{3}{*}{ Grit size } & \multicolumn{4}{|c|}{ Brand of synthetic diamonds } \\
\hline & \multicolumn{2}{|r|}{ AC 32} & \multicolumn{2}{|r|}{ AC80 } \\
\hline & $\begin{array}{l}\text { Static compressive } \\
\text { strength [N] }\end{array}$ & $\begin{array}{l}\text { Arithmetic mean of the compressive } \\
\text { strength for all grit sizes [N] }\end{array}$ & $\begin{array}{l}\text { Static compressive } \\
\text { strength [N] }\end{array}$ & $\begin{array}{l}\text { Arithmetic mean of the compressive } \\
\text { strength for all grit sizes [N] }\end{array}$ \\
\hline D107 (100/80) & 18.4 & \multirow{3}{*}{32} & 40 & \multirow{3}{*}{80} \\
\hline $\mathrm{D} 251(250 / 200)$ & 23.5 & & 78 & \\
\hline D426 (400/315) & 49.6 & & 109 & \\
\hline
\end{tabular}

Factory - Berkovitsa, Bulgaria [26]; material to be machined - hardened steel $150 \mathrm{Cr} 14$ with hardness of $64 \mathrm{HRC}$ in the shape of cylindrical workpieces with diameter $d_{w}=30 \mathrm{~mm}$ and length $L_{w}=150 \mathrm{~mm}$; method of grinding - plunge grinding; cutting speed $v_{c}=30 \mathrm{~m} / \mathrm{s}$; cutting depth $a_{e}=0.1 \mathrm{~mm}$; speed ratio $q=60$; radial infeed $f_{r}=0.01 \mathrm{~mm} / \mathrm{rev}$; coolant lubricant - sulfofresol (emulsion with $5 \%$ concentration, which is fed through a free-falling jet through an open nozzle, the flow rate being approximately $1 \mathrm{~m} / \mathrm{s}$, and the consumption - approximately $9 \mathrm{l} / \mathrm{min}$ ).

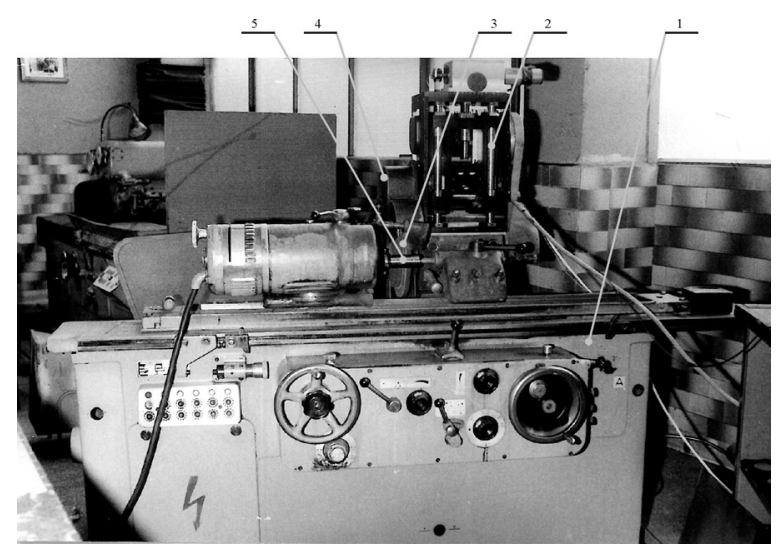

Fig. 1. Experimental setup; 1. Cylindrical grinder KUF 250/500; 2. Special attachment; 3. Grinding wheel; 4. Diamond roller dresser; 5. Workpiece

The grinding wheels are dressed using diamond roller dressers with a diameter of $92 \mathrm{~mm}$ produced by electroplating, with a layer of medium- and highstrength synthetic diamonds of AC32 and AC80 types by the Russian State Standard 9206-80 and the Ukrainian State Standard 3292-95 with grit sizes D426, D251 and D107 (Table 1) [27] to [29]. AC32 and AC80 are brands of synthetic diamond grinding powders of varying strength manufactured by the V. Bakul Institute for Superhard Materials.

The different grit sizes of the diamond roller dressers and grinding wheels provide values of the control factor $q_{g}=1.2$ to 2.56 .
In order to perform dressing with diamond roller dressers by using the plunge grinding method, a special attachment [30] has been designed. It is fixed on the grinding saddle of cylindrical grinder KUF 250/500. The control system of the attachment makes possible uni-directional and counter-directional dressing as well as variation of the control factors (radial feed rate $f_{r d}$, dressing speed ratio $q_{d}$ and dress-out time $t_{d}$ ) within the following limits: $f_{r d}=0.2 \mathrm{~mm} / \mathrm{min}$ to 1.4 $\mathrm{mm} / \mathrm{min}, q_{d}=0.2$ to $0.8, t_{d}=1 \mathrm{~s}$ to $9 \mathrm{~s}$. These conditions guarantee the quality of the machined surfaces and the lifetime of the dressing tool and dressed tool [25], [30], and [31].

The studied response variables are: the roughness $R a_{w, i h}[\mu \mathrm{m}]$ and accuracy $\delta_{w, i h}[\mu \mathrm{m}]$ of the ground surface, the grinding wheel lifetime $T_{s, i h}$ [min] and the manufacturing net costs of the grinding operation $C_{i h}[€ / \mathrm{pc}]$ (ih is the combination of the code of the synthetic diamond brand and the type of dressing, see Table 2).

The ground surface roughness, evaluated by the arithmetic mean deviation of the profile, is measured with Mitutoyo SJ-201 profilometer. The accuracy of the ground surface shape is evaluated by the complex indicator: deviation from cylindricity, employing an apparatus for measuring deviation from roundness and cylindricity Roundtest RA-114/116 of the Mitutoyo company.

Table 2. Code of the combination: brand of synthetic diamonds, dressing method'

\begin{tabular}{lcc}
\hline \multirow{2}{*}{ Dressing method, $h$} & \multicolumn{2}{c}{ Brand of synthetic diamonds, $i$} \\
\cline { 2 - 3 } & AC32 $(i=1)$ & AC80 $(i=2)$ \\
\hline Uni-directional $(h=1)$ & 11 & 21 \\
\hline Counter-directional $(h=2)$ & 12 & 22 \\
\hline
\end{tabular}

The grinding wheel lifetime corresponds to the tool operation time between two dressing events. The criterion showing the necessity of dressing is the roughness occurring on the ground surface $R a_{w}=2.5 \mu \mathrm{m}$. 
The manufacturing net costs of the grinding operation is defined as the sum of labour costs and variable additional costs including grinding wheel dressing costs, grinding wheels costs, and electric power costs. The relative shares of the manufacturing net costs components depend on the manufacturing conditions and they are not identical. The electric power costs are very rarely significant (e.g., for intensive grinding), and the grinding wheel costs are high only when the tool price is high or during operation in self-grinding mode. Therefore, it could be assumed with sufficient accuracy that the manufacturing net costs of the grinding operation is defined by the relationship:

$$
C_{i h}=C_{m, i h}+C_{d, h},
$$

where the labour costs $C_{m, i h}$ and the dressing costs $C_{d, i h}$ are defined by the following formulae:

$$
\begin{gathered}
C_{m, i h}=C_{m} \cdot t_{m, i h}, \\
C_{d, i h}=\frac{C_{m} \cdot t_{d 0}+S_{s} \cdot W_{s}+S_{d} / T_{d, i h}}{T_{s, i h}} \cdot t_{m, i h},
\end{gathered}
$$

where: $C_{m}[€ / \mathrm{min}]$ is average labour costs; $t_{m, i h}$ [min] is grinding time: $t_{m, i h}=W_{w} / Q_{w, i h}\left(W_{w}\left[\mathrm{~mm}^{3}\right]\right.$ is the volume of cut layer removed during grinding; $W_{w}=$ const at constant cutting conditions during grinding; $Q_{w, i h}\left[\mathrm{~mm}^{3} / \mathrm{min}\right]$ is the production rate of grinding process determined in [25]); $t_{d 0}[\mathrm{~min}]$ is dressing time: $t_{d 0}=a_{d} / f_{r d}+t_{d}\left(a_{d}=0.03 \mathrm{~mm}\right.$ is a depth of dressing $) ; S_{s}$ $\left[€ / \mathrm{mm}^{3}\right]$ is the cost per unit volume of the grinding wheel; $W_{s}\left[\mathrm{~mm}^{3}\right]$ is the volume of abrasive layer removed during dressing; $S_{d}[€]$ is the price of diamond roller dressers; $T_{d, i h}$ is the diamond roller dresser lifetime evaluated by the number of working runs carried out up to reaching the permissible deviation of the grinding wheel profile after dressing $\delta_{w}=0.02 \mathrm{~mm}$ (they are counted by a mechanical counter comprised into the control system of the dressing attachment).

On the basis of preliminary experimental studies conducted [30], it is assumed that the general form of the model describing the relation between the chosen response variables of fine cylindrical grinding $Y_{j}$ and the group of independent variables are the control factors $f_{r d}\left(X_{1}\right), q_{d}\left(X_{2}\right), t_{d}\left(X_{3}\right)$ and $q_{g}\left(X_{4}\right)$, is:

$$
Y_{j}=E_{j} \cdot X_{1}^{b_{1 j}} \cdot X_{2}^{b_{2 j}} \cdot X_{3}^{b_{3 j}} \cdot X_{4}^{b_{4 j}},
$$

where $Y_{1}=R a_{w, i h} ; Y_{2}=\delta_{w, i h} ; Y_{3}=T_{s, i h} ; Y_{4}=C_{i h} ; b_{1 j}, b_{2 j}$, $b_{3 j}, b_{4 j}$ are exponents that determine the magnitude and the type of effect of control factors on the studied response variable $Y_{j}$ of the grinding process; $E_{j}$ is a coefficient accounting for the effect of the control factors (kinematic cutting parameters in grinding, physical-mechanical properties of the material to be machined, shape and size of the grinding wheel, type and quantity of the coolant lubricant, etc.).

In order to build the model in Eq. (4), it is linearized by a logarithmic transformation, as follows:

$$
\begin{aligned}
\ln Y_{j}= & \ln E_{j}+b_{1 j} \ln X_{1}+b_{2 j} \ln X_{2} \\
& +b_{3 j} \ln X_{3}+b_{4 j} \ln X_{4} .
\end{aligned}
$$

Taking into account the interactions between the control factors, Eq. (5) can be written in this form:

$$
\begin{aligned}
Y_{j}^{\prime} & =b_{0, j}+\sum_{p=1}^{4} b_{p, j} A_{p}+\sum_{\substack{p=1 \\
p<t}}^{4} b_{p t, j} A_{p} A_{t} \\
& +\sum_{\substack{p=1 \\
p<<<l}}^{4} b_{p t l, j} A_{p} A_{t} A_{l}+b_{1234} A_{1} A_{2} A_{3} A_{4},
\end{aligned}
$$

where $\quad Y_{j}^{\prime}=\ln Y_{j}, \quad b_{0 j}=\ln E_{j}, \quad A_{1}=\ln X_{1}=\ln f_{r d}$, $A_{2}=\ln X_{2}=\ln q_{d}, A_{3}=\ln X_{3}=\ln t_{d}, A_{4}=\ln X_{4}=\ln q_{g}$.

Table 3. Factor levels in the experimental design

\begin{tabular}{lccccc}
\hline & \multicolumn{5}{c}{ Factors } \\
\cline { 2 - 6 } Factor levels & $\begin{array}{c}\text { Coded } \\
x_{p}\end{array}$ & $\begin{array}{c}X_{1}=f_{r d} \\
{[\mathrm{~mm} / \mathrm{min}]}\end{array}$ & $X_{2}=q_{d}$ & $\begin{array}{c}X_{3}=t_{d} \\
{[\mathrm{~s}]}\end{array}$ & $X_{4}=q_{g}$ \\
\hline lower $x_{p l}, X_{p l}$ & -1 & 0.2 & 0.2 & 1 & 1.2 \\
\hline upper $x_{p u}, X_{p u}$ & +1 & 1.4 & 0.8 & 9 & 2.56 \\
\hline basic $x_{p o}, X_{p o}$ & 0 & 0.5 & 0.4 & 3 & 1.75 \\
\hline$x_{p}=\frac{2\left(\ln X_{p}-\ln X_{p u}\right)}{\ln X_{p u}-\ln X_{p l}}+1$, & $X_{p o}=0.5\left(\ln X_{p u}+\ln X_{p l}\right)$ \\
\hline
\end{tabular}

To build model in Eq. (6), the first order design of experiment is applied, in particular a full factorial design of experiments is used. The minimum number for the levels of factor variation is two (Table 3), and the required number of runs is $N=2^{p}=2^{4}=16$ ( $p=4$ is the number of the control factors). The design of the experiments and the processing of the experimental results have been performed following the methodology presented in [32]. The models of grinding wheel lifetime $T_{s, i h}$, roughness $R a_{w, i h}$ and accuracy $\delta_{w, i h}$ of the ground surface are synthesized according to actually measured values of the response variables in fine cylindrical grinding with grinding wheels dressed under certain conditions with diamond dresser rollers with a layer of synthetic diamonds of medium and high strength. The model of the manufacturing net costs $C_{i h}$ is built on the basis of 
values calculated according to Eq. (1). The coefficient of multiple correlation $R_{j}$, the standard quadric mean deviation and the regression coefficients are determined by means of linear regression analysis. To check the significance of the regression coefficients, the model adequacy and the process description quality, the basic level (radial feed rate $f_{r d}=0.5$ $\mathrm{mm} / \mathrm{min}$; speed ratio $q_{d}=0.4$; dress-out time $t_{d}=5 \mathrm{~s}$; ratio between the grit sizes of the diamond roller dresser and the grinding wheel $q_{g}=1.85$, Table 3 ) has been selected as the most informative point, where four observations have been performed $(n=4)$.

\subsection{Experimental Results and Modelling}

The designs of the experiments with the values of the response variables of the fine cylindrical grinding process are presented in Tables 4 and 5 .

After statistical analysis of the experimental results and transformation of the independent variables from coded to natural type (Table 3), theoreticalexperimental models of roughness and accuracy of the ground surface, grinding wheel lifetime and the manufacturing net costs have been built and their general form is the following:

$$
Y_{j}=E_{j, i h} \cdot f_{r d}^{b_{1 j, i h}} \cdot q_{d}^{b_{2 j, i h}} \cdot t_{d}^{b_{j j, i h}} \cdot q_{g}^{b_{j j, i h}},
$$

The determined values of the constants $E_{j, i h}$ and exponents $b_{1, i h}, b_{2, i h}, b_{3, i h}, b_{4, i h}$ in the regression models in Eq. (7) are given in Table 6. The values of the standard deviation for each of the experiments carried out, characterizing the repeatability of the experimental results and used for determining the empirical values of the Fisher criterion $\widehat{F}_{j, i h}$ and the multiple correlation coefficient $\hat{R}_{j, i h}$, are $S_{R j, i h}^{2}=$ 0.0004 to 0.0045 . They have been determined on the basis of the four observations performed for each of the response variables of the fine grinding process at a combination of the main levels of the control factors $\left(f_{r d}=0.5 \mathrm{~mm} / \mathrm{min} ; q_{d}=0.4 ; t_{d}=5 \mathrm{~s} ; q_{g}=1.85\right)$, Tables 4 and 5 . The constructed models are adequate, which is proved by comparing the empirical $\widehat{F}_{j, i h}$ and tabular $F_{j, i h}^{t}$ values of the Fisher criterion $\left(\widehat{F}_{j, i h}<F_{j, i h}^{t}\right.$, Table 6). They describe with high accuracy the dependencies between the response variables and the control factors (the values of the coefficient of multiple correlation are $\hat{R}_{j, i h}=0.981$ to 0.998 ).

Table 4. Design of the experiment and response variables of the grinding process (in dressing with diamond roller dressers of synthetic diamonds AC32)

\begin{tabular}{|c|c|c|c|c|c|c|c|c|c|c|c|}
\hline \multirow{3}{*}{$\begin{array}{c}f_{r d} \\
{[\mathrm{~mm} / \mathrm{min}]}\end{array}$} & \multirow{2}{*}{\multicolumn{3}{|c|}{ Control factors }} & \multicolumn{8}{|c|}{ Response variables of the grinding process } \\
\hline & & & & \multicolumn{4}{|c|}{ Uni-directional dressing } & \multicolumn{4}{|c|}{ Counter-directional dressing } \\
\hline & $q_{d}$ & $\begin{array}{c}t_{d} \\
{[\mathrm{~s}]}\end{array}$ & $q_{g}$ & $\begin{array}{c}R a_{w 11} \\
{[\mu \mathrm{m}]}\end{array}$ & $\begin{array}{l}\delta_{w 11} \\
{[\mu \mathrm{m}]}\end{array}$ & $\begin{array}{l}T_{S 11} \\
{[\mathrm{~min}]}\end{array}$ & $\begin{array}{c}C_{11} \\
{[€ / p c]}\end{array}$ & $\begin{array}{c}R a_{w 12} \\
{[\mu \mathrm{m}]}\end{array}$ & $\begin{array}{l}\delta_{w 12} \\
{[\mu \mathrm{m}]}\end{array}$ & $\begin{array}{c}T_{s 12} \\
\text { [min] }\end{array}$ & $\begin{array}{c}C_{12} \\
{[€ / \mathrm{pc}]}\end{array}$ \\
\hline 0.2 & 0.2 & 1 & 1.2 & 0.86 & 5.64 & 17.02 & 0.031 & 0.69 & 5.64 & 18.60 & 0.035 \\
\hline 1.4 & 0.2 & 1 & 1.2 & 1.03 & 9.94 & 28.03 & 0.027 & 0.87 & 9.94 & 27.94 & 0.029 \\
\hline 0.2 & 0.8 & 1 & 1.2 & 1.01 & 6.06 & 23.60 & 0.028 & 0.56 & 6.06 & 9.80 & 0.050 \\
\hline 1.4 & 0.8 & 1 & 1.2 & 1.22 & 10.52 & 29.20 & 0.025 & 0.70 & 10.52 & 16.90 & 0.041 \\
\hline 0.2 & 0.2 & 9 & 1.2 & 0.67 & 4.14 & 13.97 & 0.037 & 0.58 & 4.14 & 11.30 & 0.052 \\
\hline 1.4 & 0.2 & 9 & 1.2 & 0.81 & 7.28 & 22.25 & 0.033 & 0.73 & 7.28 & 20.10 & 0.036 \\
\hline 0.2 & 0.8 & 9 & 1.2 & 0.79 & 3.54 & 19.30 & 0.036 & 0.47 & 3.54 & 8.20 & 0.056 \\
\hline 1.4 & 0.8 & 9 & 1.2 & 0.95 & 6.15 & 23.84 & 0.032 & 0.59 & 6.15 & 12.90 & 0.051 \\
\hline 0.2 & 0.2 & 1 & 2.56 & 0.41 & 8.42 & 38.80 & 0.022 & 0.34 & 8.42 & 42.20 & 0.022 \\
\hline 1.4 & 0.2 & 1 & 2.56 & 0.47 & 11.39 & 56.20 & 0.019 & 0.41 & 11.39 & 68.00 & 0.019 \\
\hline 0.2 & 0.8 & 1 & 2.56 & 0.45 & 9.72 & 46.30 & 0.019 & 0.25 & 9.72 & 22.60 & 0.036 \\
\hline 1.4 & 0.8 & 1 & 2.56 & 0.58 & 12.2 & 65.00 & 0.017 & 0.30 & 12.2 & 35.70 & 0.023 \\
\hline 0.2 & 0.2 & 9 & 2.56 & 0.29 & 6.17 & 28.80 & 0.034 & 0.26 & 6.17 & 26.70 & 0.039 \\
\hline 1.4 & 0.2 & 9 & 2.56 & 0,32 & 8.34 & 43.70 & 0.029 & 0,31 & 8.34 & 39.10 & 0.028 \\
\hline 0.2 & 0.8 & 9 & 2.56 & 0.34 & 5.68 & 36.10 & 0.030 & 0.20 & 5.68 & 20.90 & 0.047 \\
\hline 1.4 & 0.8 & 9 & 2.56 & 0.41 & 7.13 & 46.00 & 0.026 & 0.23 & 7.13 & 30.40 & 0.034 \\
\hline 0.5 & 0.4 & 3 & 1.85 & 0.62 & 7.33 & 33.5 & 0.026 & 0.41 & 7.33 & 26.9 & 0.035 \\
\hline 0.5 & 0.4 & 3 & 1.85 & 0.6 & 7.4 & 33.4 & 0.024 & 0.4 & 7.4 & 26.8 & 0.033 \\
\hline 0.5 & 0.4 & 3 & 1.85 & 0.58 & 6.8 & 32.5 & 0.025 & 0.45 & 6.8 & 25.6 & 0.0324 \\
\hline 0.5 & 0.4 & 3 & 1.85 & 0.55 & 7.25 & 32.1 & 0.0248 & 0.47 & 7.25 & 24.7 & 0.0342 \\
\hline
\end{tabular}


Table 5. Design of the experiment and response variables of the grinding process (in dressing with diamond roller dressers of synthetic diamonds AC80)

\begin{tabular}{|c|c|c|c|c|c|c|c|c|c|c|c|}
\hline \multirow{2}{*}{\multicolumn{4}{|c|}{ Control factors }} & \multicolumn{8}{|c|}{ Response variables of the grinding process } \\
\hline & & & & \multicolumn{4}{|c|}{ Uni-directional dressing } & \multicolumn{4}{|c|}{ Counter-directional dressing } \\
\hline$\underset{[\mathrm{mm} / \mathrm{min}]}{f_{r d}}$ & $q_{d}$ & $\begin{array}{l}t_{d} \\
{[\mathrm{~s}]}\end{array}$ & $q_{g}$ & $\begin{array}{c}R a_{w 21} \\
{[\mu \mathrm{m}]}\end{array}$ & $\begin{array}{l}\delta_{w 21} \\
{[\mu \mathrm{m}]}\end{array}$ & $\begin{array}{c}T_{s 21} \\
{[\mathrm{~min}]}\end{array}$ & $\begin{array}{c}C_{21} \\
{[€ / \mathrm{pc}]}\end{array}$ & $\begin{array}{c}R a_{w 22} \\
{[\mu \mathrm{m}]}\end{array}$ & $\begin{array}{l}\delta_{w 22} \\
{[\mu \mathrm{m}]}\end{array}$ & $\begin{array}{r}T_{s 22} \\
{[\mathrm{~min}]}\end{array}$ & $\begin{array}{r}C_{22} \\
{[€ / p c]}\end{array}$ \\
\hline 0.2 & 0.2 & 1 & 1.2 & 1.21 & 5.64 & 24.06 & 0.031 & 1.08 & 5.64 & 23.70 & 0.035 \\
\hline 1.4 & 0.2 & 1 & 1.2 & 1.44 & 9.94 & 25.70 & 0.027 & 1.28 & 9.94 & 25.10 & 0.029 \\
\hline 0.2 & 0.8 & 1 & 1.2 & 1.31 & 6.06 & 26.80 & 0.029 & 0.95 & 6.06 & 11.80 & 0.050 \\
\hline 1.4 & 0.8 & 1 & 1.2 & 1.54 & 10.52 & 31.50 & 0.026 & 1.13 & 10.52 & 16.20 & 0.041 \\
\hline 0.2 & 0.2 & 9 & 1.2 & 0.88 & 4.14 & 16.50 & 0.039 & 0.73 & 4.14 & 10.80 & 0.052 \\
\hline 1.4 & 0.2 & 9 & 1.2 & 1.05 & 7.28 & 20.00 & 0.033 & 0.86 & 7.28 & 17.80 & 0.037 \\
\hline 0.2 & 0.8 & 9 & 1.2 & 0.97 & 3.54 & 20.30 & 0.037 & 0.64 & 3.54 & 8.30 & 0.055 \\
\hline 1.4 & 0.8 & 9 & 1.2 & 1.12 & 6.15 & 22.90 & 0.033 & 0.76 & 6.15 & 12.80 & 0.051 \\
\hline 0.2 & 0.2 & 1 & 2.56 & 0.64 & 8.42 & 58.00 & 0.022 & 0.62 & 8.42 & 52.50 & 0.022 \\
\hline 1.4 & 0.2 & 1 & 2.56 & 0.72 & 11.39 & 67.00 & 0.019 & 0.68 & 11.39 & 75.00 & 0.019 \\
\hline 0.2 & 0.8 & 1 & 2.56 & 0.68 & 9.72 & 63.00 & 0.019 & 0.56 & 9.72 & 36.40 & 0.035 \\
\hline 1.4 & 0.8 & 1 & 2.56 & 0.77 & 12.2 & 78.00 & 0.017 & 0.61 & 12.2 & 50.00 & 0.022 \\
\hline 0.2 & 0.2 & 9 & 2.56 & 0.36 & 6.17 & 29.10 & 0.034 & 0.35 & 6.17 & 26.90 & 0.039 \\
\hline 1.4 & 0.2 & 9 & 2.56 & 0,48 & 8.34 & 39.30 & 0.029 & 0.46 & 8.34 & 35.90 & 0.028 \\
\hline 0.2 & 0.8 & 9 & 2.56 & 0.46 & 5.68 & 34.70 & 0.030 & 0.30 & 5.68 & 29.10 & 0.046 \\
\hline 1.4 & 0.8 & 9 & 2.56 & 0.55 & 7.13 & 57.00 & 0.027 & 0.37 & 7.13 & 33.30 & 0.034 \\
\hline 0.5 & 0.4 & 3 & 1.85 & 0.89 & 7.33 & 41.7 & 0.026 & 0.7 & 7.33 & 26.4 & 0.035 \\
\hline 0.5 & 0.4 & 3 & 1.85 & 0.8 & 7.4 & 40.1 & 0.024 & 0.68 & 7.4 & 29.3 & 0.033 \\
\hline 0.5 & 0.4 & 3 & 1.85 & 0.81 & 6.8 & 38.8 & 0.025 & 0.65 & 6.8 & 29.5 & 0.032 \\
\hline 0.5 & 0.4 & 3 & 1.85 & 0.85 & 7.25 & 38 & 0.0245 & 0.6 & 7.25 & 26.5 & 0.034 \\
\hline
\end{tabular}

Table 6. Values of constants and exponents in the theoretical-experimental models, Eq. (7)

\begin{tabular}{|c|c|c|c|c|c|c|c|}
\hline \multirow{2}{*}{$\begin{array}{c}\text { Response } \\
\text { variables }\end{array}$} & \multirow{2}{*}{$\begin{array}{c}\text { Constants } \\
\qquad E_{j, i h}\end{array}$} & \multirow[b]{2}{*}{$b_{1 j, i h}$} & \multicolumn{2}{|l|}{ Exponents } & \multirow[b]{2}{*}{$b_{4 j, i h}$} & \multicolumn{2}{|c|}{ Fisher criterion } \\
\hline & & & $b_{2 j, i h}$ & $b_{3 j, i h}$ & & $\widehat{F}_{j, i h}$ & $F_{j, i h}^{t}$ \\
\hline$R a_{w 11}$ & 1.503 & 0.091 & 0.123 & -0.133 & -1.077 & 0.613 & 8.765 \\
\hline$R a_{w 12}$ & 0.787 & 0.103 & -0.183 & -0.099 & -1.084 & 0.442 & 8.765 \\
\hline$R a_{w 21}$ & 1.909 & 0.088 & 0.074 & -0.169 & -0.959 & 1.292 & 8.765 \\
\hline$R a_{w 22}$ & 1.285 & 0.086 & -0.098 & -0.209 & -0.853 & 0.650 & 8.765 \\
\hline $\begin{array}{l}\delta_{w 11} \\
\delta_{w 12} \\
\delta_{w 21} \\
\delta_{w 22} \\
\end{array}$ & 9.541 & $0.324-0.199 A_{4}$ & $0.082-0.199 A_{3}$ & -0.261 & 0.255 & 0.535 & 8.785 \\
\hline$T_{s 11}$ & 23.810 & $0.068-0.117 A_{2}+0.083 A_{4}$ & $0.052+0.058 A_{4}+0.091 A_{1} A_{4}$ & $-0.087-0.041 A_{4}$ & 1.029 & 2.695 & 8.845 \\
\hline$T_{s 12}$ & 11.296 & 0.236 & $-0.435+0.095 A_{3}$ & -0.057 & 1.091 & 2.827 & 8.875 \\
\hline$T_{s 21}$ & 25.376 & $0.017+0.031 A_{3}+0.104 A_{4}$ & 0.130 & $-0.095-0.136 A_{4}$ & 1.254 & 3.764 & 8.845 \\
\hline$T_{s 22}$ & 10.833 & 0.155 & $-0.472+0.108 A_{3}+0.229 A_{4}$ & -0.114 & 1.524 & 2.530 & 8.812 \\
\hline$C_{11}$ & 0.029 & -0.068 & -0.055 & $0.080+0.130 A_{4}$ & -0.509 & 1.343 & 8.785 \\
\hline$C_{12}$ & 0.049 & $\begin{array}{l}-0.120+0.017 A_{2}+ \\
+0.047 A_{3}-0.121 A_{4} \\
\end{array}$ & $\begin{array}{l}0.264-0.017 A_{3}-0.084 A_{4} \\
+0.052 A_{1} A_{3}-0.132 A_{1} A_{4} \\
\end{array}$ & $-0.071+0.104 A_{4}$ & -0.675 & 1.506 & 8.887 \\
\hline$C_{21}$ & 0.027 & -0.067 & -0.066 & $0.076+0.130 A_{4}$ & -0.484 & 1.028 & 8.785 \\
\hline$C_{22}$ & 0.048 & $\begin{array}{l}-0.116+0.017 A_{2}+ \\
+0.048 A_{3}-0.122 A_{4} \\
\end{array}$ & $\begin{array}{l}0.248-0.014 A_{3}-0.085 A_{4} \\
+0.053 A_{1} A_{3}-0.133 A_{1} A_{4}\end{array}$ & $0.076+0.109 A_{4}$ & -0.693 & 1.531 & 8.887 \\
\hline & & $A_{1}=\ln$ & $d ; A_{2}=\ln q_{d} ; A_{3}=\ln t_{d} ; A$ & $\ln q_{g}$ & & & \\
\hline
\end{tabular}




\subsection{Analysis of the Experimental Results}

The analysis of the theoretical-experimental models in Eq. (7) and the graphics plotted on the basis of them (Fig. 2) allows the following conclusions to be drawn:

(1) The studied response variables of the fine grinding process greatly depend on the diamond roller dresser grit size/the grinding wheel grit size ratio.

(1.1) When $q_{g}$ increases within the studied range (2.1 times), the grinding wheel lifetime increases (by 1.8 to 3 times). The impact of $q_{g}$ depends on the types of synthetic diamonds in the working layer of the diamond roller dressers, the conditions and direction of dressing, and it is most strongly marked in counter-directional dressing with diamond roller dressers with a working layer of synthetic diamonds AC 80 .

(1.2) The roughness of the ground surface decreases (by 1.9 to 2.3 times) when $q_{g}$ increases. The impact of $q_{g}$ is more strongly marked in counterdirectional dressing with diamond roller dressers of synthetic diamonds AC32. The decrease in the ground surface roughness with an increase in $q_{g}$ is related to an improvement of the grinding wheel cutting ability, as well as to a decrease in forces and temperature load in the cutting zone in grinding.

(1.3) The manufacturing net costs of the grinding operation decrease (by 1.1 to 1.7 times) with an increase in the grit ratio $q_{g}$. The impact of $q_{g}$ is the greatest in counter-directional dressing with diamond roller dressers with a working layer of synthetic diamonds AC80 and it increases when dress-out time $t_{d}$ decreases.

(1.4) When the grit ratio $q_{g}$ increases, the ground surface accuracy decreases (deviation from cylindricity rises by 1.15 to 1.55 times), and the impact is identical in uni-directional and counterdirectional dressing with diamond roller dressers of medium- and high-strength synthetic diamonds AC32 and AC80. The impact of $q_{g}$ decreases with an increase in radial feed rate. The relatively small impact of $q_{g}$ on the ground surface accuracy is determined by the impact of this factor, different in character and rate, on the grinding wheel macro-geometry and on the normal cutting force in grinding, and by their key role for the ground surface accuracy, established in [30].

(2) The direction of dressing has different influence on the response variables of the fine grinding process.
(2.1) The uni-directional dressing ensures longer lifetime of the grinding wheels (up to 2.5 times) compared to counter-directional dressing. The difference in the lifetimes of the grinding wheels dressed uni-directionally and counterdirectionally grows with an increase in radial feed rate $f_{r d}$ and in dressing speed ratio $q_{d}$, and with a decrease in the grit ratio $q_{g}$. This tendency is valid for dressing with diamond roller dressers with a working layer of synthetic diamonds $\mathrm{AC} 32$ and $\mathrm{AC} 80$ and it is related to the greater "roughness" of the cutting surface of the grinding wheels after their uni-directional dressing [30], [33] and [34] as well as with the destruction of the wheel structure as an additional consideration [35] and [36].

(2.2) The roughness of the machined surface after grinding with grinding wheels dressed counterdirectionally is smaller (up to 1.9 times) compared to the roughness after grinding with grinding wheels dressed uni-directionally. This tendency can be explained by the lower "roughness" of the cutting surface of the grinding wheels dressed counter-directionally [30], [33] and [34] and it is more strongly marked in dressing with diamond roller dressers of synthetic diamonds AC 32 .

(2.3) The accuracy of the ground surface does not depend on the direction of dressing and the type of synthetic diamonds in the working layer of the diamond roller dressers. This is related to the fact that the grinding wheel macro-geometry affects directly the ground surface accuracy, as the experimentally measured values of radial run-out of grinding wheels of different specifications after uni-directional and counter-directional dressing with diamond roller dressers with a working layer of medium- and high-strength synthetic diamonds AC32 and AC80 differ by not more than $5 \%$ [30].

(2.4) The manufacturing net costs of grinding with grinding wheels dressed uni-directionally are lower (up to 1.8 times) than the costs in grinding with tools dressed counter-directionally. The difference in manufacturing net costs values rises with an increase in speed ratio $q_{d}$ and with a decrease in grit ratio $q_{g}$ and it does not depend on the type of synthetic diamonds in the working layer of the diamond roller dressers.

(3) The dressing conditions have an effect different in character and rate on the response variables in the fine grinding process, which depends on the method of dressing and the type of synthetic diamonds in the working layer of the diamond roller dressers. 


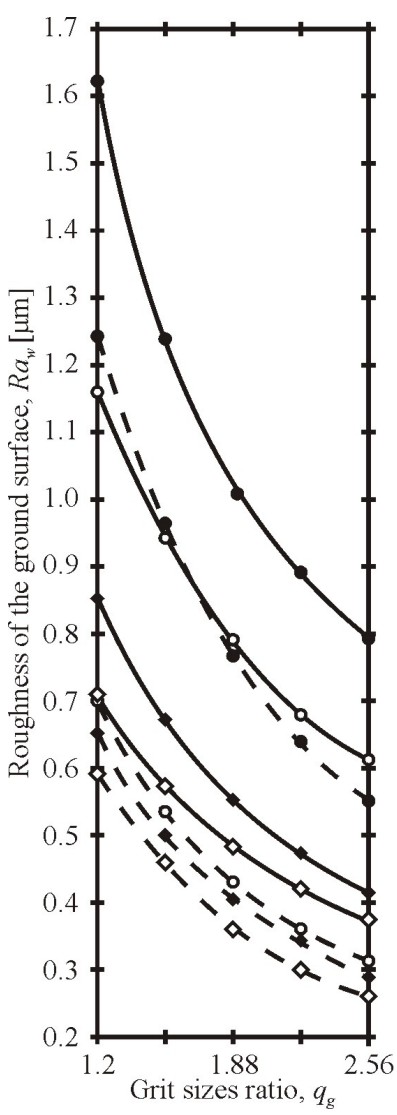

a)

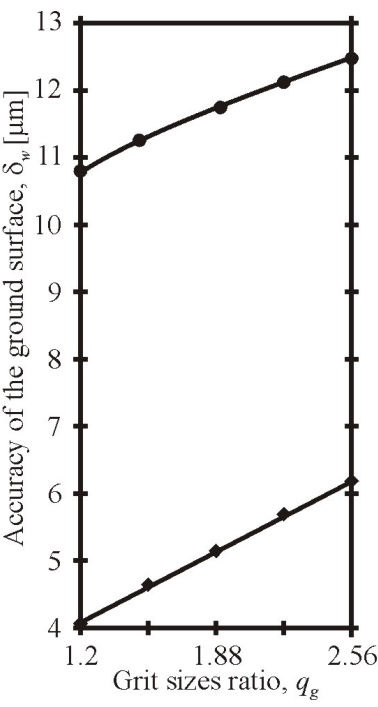

b)

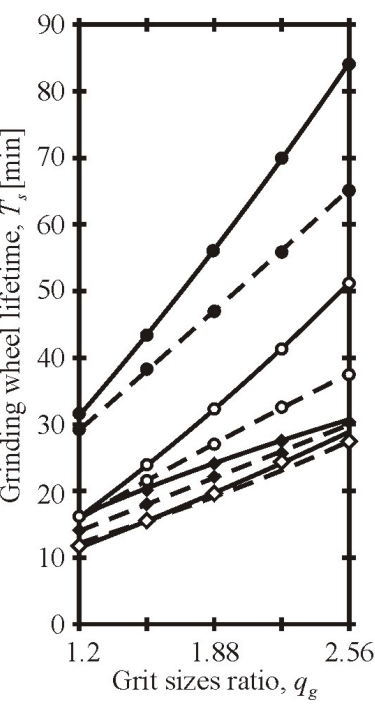

c)

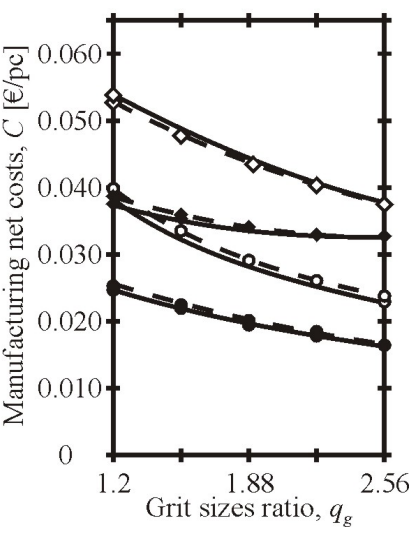

d)

Fig. 2. Impact of the dressing system parameters on: a) roughness of the ground surface, b) accuracy of the ground surface, c) grinding wheel lifetime, and d) manufacturing net costs of grinding operation

(3.1.) The increase in radial feed rate in dressing leads to a respective increase in the grinding wheels lifetime (up to 1.6 times) and in the ground surface roughness (up to 1.3 times) and to a decrease in the manufacturing net costs (up to 1.5 times). The influence of $f_{r d}$ is most strongly marked in counter-directional dressing with diamond roller dressers with a working layer of synthetic diamonds AC32.

With an increase in radial feed rate in dressing the ground surface accuracy decreases (by $30 \%$ to 74 $\%$ ) depending on the grit ratio $q_{g}$. The impact of $f_{r d}$ rises with a decrease in $q_{g}$. This character of change is explained by an increase in the area of the removed abrasive layer, as well as an increase in the forces and heat which results in quality deterioration of the grinding wheel profiled surface and in a decrease in ground surface accuracy, respectively [10] and [30].

(3.2) The speed ratio impact in dressing on the grinding wheel lifetime is most strongly pronounced in counter-directional dressing with diamond roller dressers with a working layer of synthetic diamonds AC32, as a decrease in $q_{d}$ leads to a respective increase in the grinding wheel lifetime (up to $83 \%$ ). The tendency has an opposite character in uni-directional dressing of grinding wheels.

The speed ratio $q_{d}$ has a different impact on the roughness of the ground surface and the manufacturing net costs of the grinding operation depending on the method of dressing. In unidirectional dressing, when $q_{d}$ increases, the ground surface roughness increases, and the manufacturing net costs decrease. In counterdirectional dressing, the increase in $q_{d}$ leads to a respective decrease in roughness and an increase in manufacturing net costs. The speed ratio impact is most strongly marked in counterdirectional dressing with diamond roller dressers of synthetic diamonds AC32, and in the studied variation range of $q_{d}$ the decrease in roughness is 
up to $23 \%$, and the increase in manufacturing net costs is up to $67 \%$.

Of all dressing conditions the speed ratio $q_{d}$ has the least influence on the ground surface accuracy. When $q_{d}$ increases, the deviation from cylindricity increases or decreases (up to $12 \%$ ) depending on the dress-out time $t_{d}$.

(3.3) With a decrease in dress-out time $t_{d}$ the grinding wheels lifetime rises (up to $88 \%$ ). The impact of $t_{d}$ is most strongly marked in counter-directional dressing with diamond roller dressers of synthetic diamonds AC80.

Of all dressing conditions the dress-out time has the greatest effect on the roughness of the ground surface and the manufacturing net costs of the grinding operation, which is related to the mechanisms in the generation of grinding wheel topography by dressing and the occurrence of structure damage from dressing [35] and [36]. With an increase in $t_{d}$ within the studied range roughness decreases (up to $37 \%$ ), and the manufacturing net costs increase (up to $57 \%$ ). The tendency is valid for uni-directional and counter-directional dressing of grinding wheels with diamond roller dressers of medium- and high-strength synthetic diamonds. The influence of $t_{d}$ on the roughness of the ground surface is the strongest in counterdirectional dressing with diamond roller dressers of synthetic diamonds AC80. The variation rate of manufacturing net costs depending on the dress-out time is most strongly pronounced in unidirectional dressing with diamond roller dressers of synthetic diamonds $\mathrm{AC} 80$ and rises with an increase in the grit ratio $q_{g}$.

With an increase in the dress-out time $t_{d}$ the accuracy of the machined surface improves (the deviation from cylindricity decreases by 1.36 to 1.71 times). The impact of $t_{d}$ rises with an increase in the speed ratio $q_{d}$.

\section{OPTIMIZATION OF THE DRESSING SYSTEM PARAMETERS IN FINE CYLINDRICAL GRINDING}

\subsection{A Method for Optimization}

Each of the studied response variables of the fine grinding process is of certain importance but it is not sufficient for the optimum process control. The optimum values of the various response variables will be obtained by different combinations of values of the control factors (dressing conditions, type, and specification of the dressing tool), provided the cutting conditions in grinding are constant and have been assumed in the capacity of constant factors. Therefore, optimization by one response variable is not advisable. The multi-objective optimization offers a larger amount of information in order to make a well-founded decision about choosing optimum dressing system parameters. Various algorithms for carrying out optimization exist which differ in type and number of response variables as well as in the method of finding the optimum solution [37] to [39]. The existing approaches to the multiobjective optimization can be classified into three main categories [40] to [43]. The first group comprises approaches which employ the most important response variable as an objective function, and the remaining response variables are considered constraints. The major disadvantage of these approaches is that they do not implement the principal idea of multi-objective optimization, namely: all response variables to be considered simultaneously. The proposed procedures of this category would generally result in unrealistic solutions, especially when conflicting objectives are presented. In addition, the selection of one of the response variables as an objective function may not be easy in many cases. When applying the methods of the second group a region of interest is formed in which the various response variables meet certain requirements. This approach works well when there is a small number of control factors ( 2 or 3 ) and response variables (up to three). The third group consists of approaches that combine the multiple response variables into a single generalized objective function, and the multi-objective optimization problem is solved as a single-objective one. The most popular of these approaches are defined as: utility function, desirability function, loss function, distance function, and proportion of conformance.

To determine the optimum dressing system parameters in fine cylindrical grinding, the method of generalized utility function has been chosen. It is one of the most frequently used in industry methods for multi-objective optimization [42] to [44]. It is based on the idea that the quality of a product or a process that has multiple response variables is completely unacceptable if one of the response variables lies beyond the utility limits. This method determines the result as a combination of response variables and selects a set of factors for which the result is the maximum. The utility function is a scale-invariant index that enables response variables of different units of measurement to be compared. With this method, the researcher can easily determine the optimum parameters in the group of solutions. The generalized utility function has a lot of advantages over other combining methods mainly due to its flexibility, 
since it allows some of the response variables to be maximized and, at the same time, others to be minimized.

The generalized utility function is a complex indicator characterizing the response variables of fine grinding (roughness $R a_{w, i h}$ and accuracy $\delta_{w, i h}$ of the ground surface, grinding wheel lifetime $T_{s, i h}$ and manufacturing net costs of the grinding operation $\left.C_{i h}\right)$. It can be defined as geometric mean $\Phi_{G, i h}$ or arithmetic mean $\Phi_{A, i h}$ value of the utility coefficients $\eta_{j, i h}$, obtained by transforming the response variables of the fine grinding process into dimensionless quantities [32] and [40]. To solve the specific optimization problem the geometric-mean generalized utility function $\Phi_{G, i h}$ has been chosen in the capacity of optimizing parameter, since if one of the response variables of the fine grinding process does not meet the requirements for utility limits, $\Phi_{G, i h}=0$. In this case, the arithmetic-mean generalized utility function $\Phi_{A, i h} \neq 0$, and it can have a maximum value, but the dressing conditions, under which this value of $\Phi_{A, i h}$ has been obtained, are not optimal.

The solution to the optimization problem is reduced to determining a combination between the type of dressing (uni-directional or counterdirectional), the dressing conditions (radial feed rate $f_{r d}$, dressing speed ratio $q_{d}$, dress-out time $t_{d}$ ), and the specifications of the diamond roller dresser and the grinding wheel (type of synthetic diamonds and grit sizes ratio $q_{g}$ ), for which the geometric-mean generalized utility function has a maximum.

\subsection{Modelling of the Generalized Utility Function}

In order to solve the optimization problem, mathematical models have been built for defining the geometric-mean generalized utility function depending on the control factors of the dressing process. The general form of the models, based on the performed analysis of the impact of the dressing system parameters on the response variables of the fine grinding process, is:

$$
\begin{aligned}
\Phi_{G, i h}= & D_{0, i h}+\sum_{p=1}^{4} D_{p, i h} X_{p}+\sum_{p=1}^{4} D_{p p, i h} X_{p}^{2}+ \\
& \sum_{\substack{p=1 \\
p<t}}^{4} D_{p t, i h} X_{p} X_{t}+\sum_{\substack{p=1 \\
p<t<l}}^{4} D_{p t l, i h} X_{p} X_{t} X_{l}+ \\
& D_{1234, i h} X_{1} X_{2} X_{3} X_{4} .
\end{aligned}
$$

The models, Eq. (8), under the conditions of uni-directional and counter-directional dressing with diamond roller dressers of medium- and high-strength synthetic diamonds AC32 and AC80 have been created on the basis of the results from the conducted experiments following an optimum plan with the number of experiments $N=2^{p}+2 p+1=2^{4}+2 \cdot 4+1=25$ ( $p=4$ is the number of control factors), Table 7 .

In each experiment, the generalized utility function is determined as geometric-mean $\Phi_{G, i h}$ value of the particular utility functions $\eta_{j, i h}$ according to the relationship [32] and [40]:

$$
\Phi_{G, i h}=\sqrt[4]{\prod_{j=1}^{4} \eta_{j, i h}}=\sqrt[4]{\prod_{j=1}^{4} \frac{k_{j}\left(Y_{j, i h}-Y_{j u}\right)}{\Delta Y_{j}}},
$$

where $j=1$ to 4 is the number of response variables of the fine grinding process; $k_{j}$ is the utility coefficient; $k_{j}=+1$, when the increase in the response variable $Y_{j}$ is useful; $k_{j}=-1$, when the decrease in $Y_{j}$ is useful; $Y_{j u}$ is the most useless result of the response variable $Y_{j}$, obtained within the limits of the permissible space; $\Delta Y_{j}=Y_{j \max }-Y_{j \min } ; Y_{j \max }$ and $Y_{j \min }$ are utility limits (maximum and minimum values of the response variable $Y_{j}$ ).

The values of the most useless result and of the utility limits of the response variables of fine grinding are determined according to the equalities:

$$
\begin{aligned}
& R a_{w_{u}}=\left(R a_{w, i h}\right)_{\max } ; \delta_{w_{u}}=\left(\delta_{w, i h}\right)_{\max } ; T_{s_{u}}=\left(T_{s, i h}\right)_{\min } ; \\
& C_{u}=\left(C_{i h}\right)_{\max } ; \Delta R a_{w}=\left(R a_{w, i h}\right)_{\max }-\left(R a_{w, i h}\right)_{\min } ; \\
& \Delta \delta_{w}=\left(\delta_{w, i h}\right)_{\max }-\left(\delta_{w, i h}\right)_{\min } ; \Delta T_{s}=\left(T_{s, i h}\right)_{\max }-\left(T_{s, i h}\right)_{\min } ; \\
& \Delta C_{s}=\left(C_{i h}\right)_{\text {max }}-\left(C_{i h}\right)_{\min } ; \text { where }\left(R a_{w, i h}\right)_{\min }, \\
& \left(R a_{w, i h}\right)_{\max },\left(T_{s, i h}\right)_{\text {min }},\left(T_{s, i h}\right)_{\max },\left(C_{i h}\right)_{\min },\left(C_{i h}\right)_{\text {max }}
\end{aligned}
$$

are respectively the minimum and maximum values of the studied response variables of the fine grinding process (roughness, accuracy, grinding wheel lifetime and manufacturing net costs of the grinding operation), determined by the regression models, Eq. (7).

On the basis of the determined values of the generalized utility function (Table 7), applying the regression analysis method and the software product QstatLab [45], the models in Eq. (8) of the generalized utility function have been built.

The coefficients $D_{0, i h}, D_{p, i h}, D_{p p, i h}, D_{p t, i h}, D_{p t l, i h}$, and $D_{1234, \text { ih }}$ in the regression equations, Eq. (8), for $\Phi_{G, i h}$, the calculated $\widehat{F}_{i h}$ and tabular $F_{i h}^{t}=F_{i h(\alpha, v 1, v 2)}$ values of the Fisher criterion $(\alpha=0.05$ is the significance level; $v_{1}=k-1$ and $v_{2}=N-k$ are degrees of freedom; $k$ is the number of coefficients in the model), as well as the values of the determination coefficient $\hat{R}_{i h}^{2}$ are presented in Table 8. The regression models are adequate since the condition $\widehat{F}_{i h}>F_{i h}^{t}$ has been met 
Table 7. Design of the experiment and generalized utility function during uni-directional and counter-directional dressing with diamond roller dressers with a working layer of synthetic diamonds AC32 and AC80

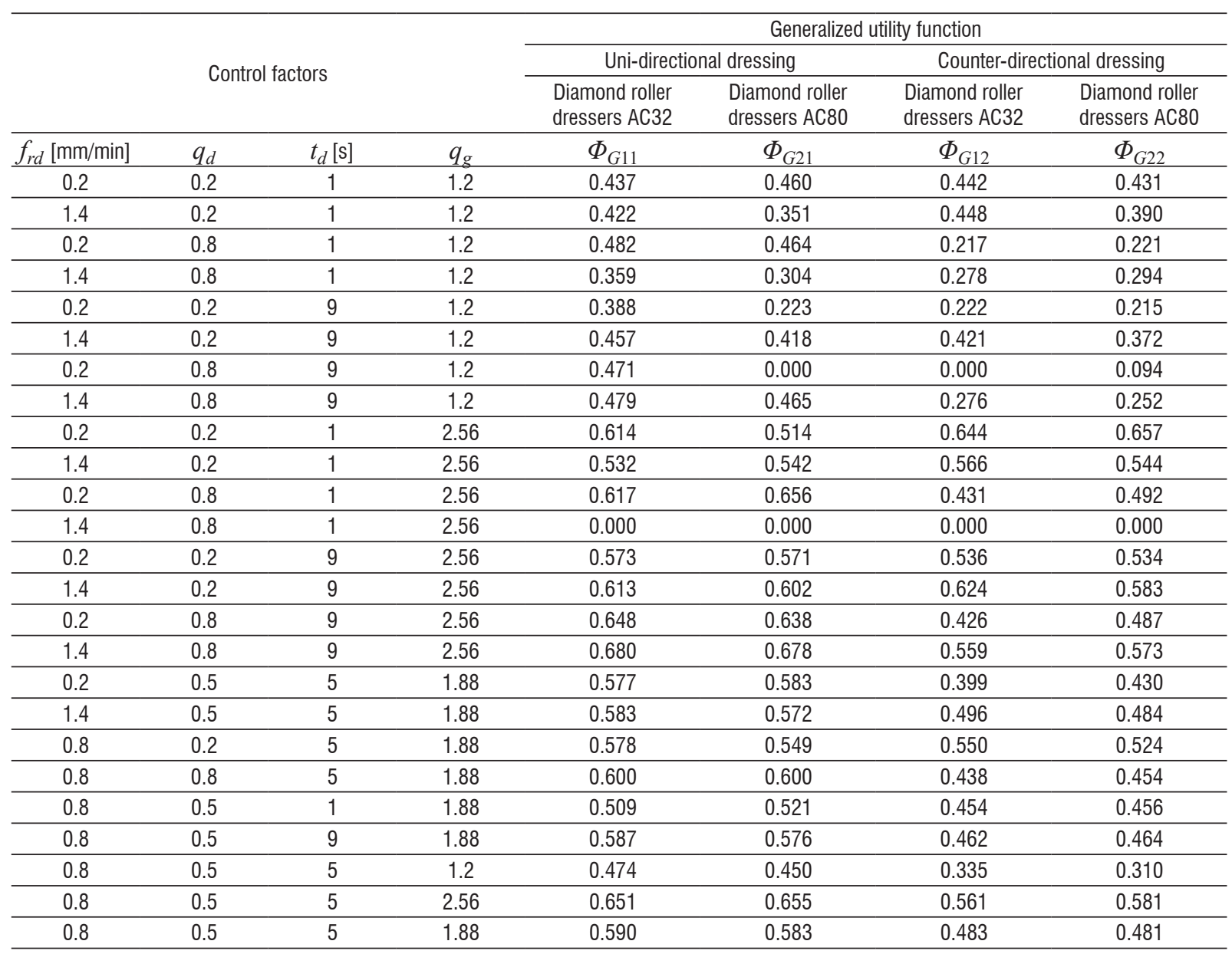

with a confidence level of $95 \%$. To determine the effect of the control factors on the generalized utility function, analysis of variance (ANOVA) has been conducted.

It has been found that of all studied factors the influence of the grit ratio is the strongest and with an increase in $q_{g}$ the function $\Phi_{G, i h}$ increases. The impact of $q_{g}$ depends upon the type of synthetic diamonds in the working layer of the diamond roller dressers, the direction and the conditions of dressing. It is most strongly pronounced in uni-directional dressing with diamond roller dressers with synthetic diamonds AC80 and grows with an increase in the speed ratio $q_{d}$ and the dress-out time $t_{d}$ and a decrease in the radial feed rate $f_{r d}$ (Figs. 3 and 4 ).

The dressing conditions have an impact different in character and rate on the geometric-mean generalized utility function, which depends on the type of synthetic diamonds in the working layer of the diamond roller dressers and the dressing method (Figs. 3 and 4). The greatest impact is with the speed ratio in uni-directional dressing with diamond roller dressers of synthetic diamonds AC80; with an increase in $q_{d}$, the generalized utility function decreases.

\subsection{Determination of Optimum Dressing System Parameters}

The optimization task has been solved during unidirectional and counter-directional dressing of grinding wheels of aluminium oxide with diamond roller dressers with working layer of medium- and high-strength synthetic diamonds AC 32 and AC 80 by applying genetic algorithm [46] and using the software product QStatLab [45].

The determined optimum dressing system parameters (type of dressing, radial feed rate of diamond roller dresser $f_{r d}$, dressing speed ratio $q_{d}$, 
Table 8. Regression coefficients and statistical analysis of regression models, Eq. (8)

\begin{tabular}{|c|c|c|c|c|}
\hline \multirow{2}{*}{$\begin{array}{l}\text { Regression } \\
\text { coefficients }\end{array}$} & \multicolumn{4}{|c|}{ Generalized utility function $\Phi_{G, i h}$} \\
\hline & $\Phi_{G 11}$ & $\Phi_{G 21}$ & $\Phi_{G 12}$ & $\Phi_{G 22}$ \\
\hline$D_{0, i h}$ & -0.110 & 0.182 & -0.582 & -0.222 \\
\hline$D_{1, i h}$ & +0.060 & - & - & - \\
\hline$D_{2, i h}$ & +0.146 & - & +0.287 & - \\
\hline$D_{3, i h}$ & +0.029 & - & - & - \\
\hline$D_{4, i h}$ & +0.514 & +0.190 & +1.142 & +0.641 \\
\hline$D_{11, i h}$ & - & - & - & - \\
\hline$D_{22, i h}$ & - & -0.297 & - & -0.231 \\
\hline$D_{33, i h}$ & -0.004 & -0.004 & - & -0.002 \\
\hline$D_{44, i h}$ & -0.097 & - & -0.271 & -0.109 \\
\hline$D_{12, i h}$ & +0.245 & +0.501 & - & +0.565 \\
\hline$D_{13, i h}$ & - & +0.034 & - & - \\
\hline$D_{23, i h}$ & - & - & -0.159 & - \\
\hline$D_{34, i h}$ & - & +0.010 & - & - \\
\hline$D_{123, i h}$ & -0.031 & -0.062 & +0.091 & -0.046 \\
\hline$D_{124, i h}$ & -0.398 & -0.394 & -0.286 & -0.440 \\
\hline$D_{134, i h}$ & - & -0.012 & - & - \\
\hline$D_{234, i h}$ & - & - & -0.286 & - \\
\hline$D_{1234, i h}$ & +0.044 & +0.053 & - & +0.049 \\
\hline $\begin{array}{r}\text { Determination } \\
\text { coefficient } \hat{R}_{i h}^{2}\end{array}$ & 0.973 & 0.965 & 0.918 & 0.953 \\
\hline \multirow{2}{*}{$\begin{array}{l}\text { Fisher } \\
\text { criterion }\end{array}$} & 50.740 & 38.981 & 27.367 & 40.344 \\
\hline & 2.602 & 2.602 & 2.614 & 2.591 \\
\hline
\end{tabular}

dress-out time $t_{d}$, diamond roller dresser grit size/ grinding wheel grit size ratio $q_{g}$ and type of synthetic diamonds), whereat the generalized utility function $\Phi_{G, i h}$ has a maximum, are presented in Table 9. Under the predicted optimum dressing system parameters, confirmation run experiments have been performed, in which the roughness and accuracy of the ground surface, the grinding wheel lifetime and the manufacturing net costs of the grinding operation have been determined. A comparison between the experimental and the predicted according to the models, Eq. (7), values of the grinding process response variables (see Table 10) has been made. The results show that the error percentage is within the permissible limits $(\leq 5 \%)$, and it is as follows: $0.65 \%$ to $5 \%$ for the roughness of the ground surface; $1.24 \%$ to $4.65 \%$ for the accuracy of the ground surface; $1.83 \%$ to $3.91 \%$ for the grinding wheel lifetime; $0.37 \%$ to $5 \%$ for the manufacturing net costs of the grinding operation. These results prove that the recommended dressing system parameters are optimum and correct.

The analysis of the obtained results shows that during uni-directional and counter-directional dressing with diamond roller dressers of synthetic diamonds AC32 and AC80 the maximum values of the generalized utility function are obtained for different combinations of speed ratio $q_{d}$, radial feed

Table 9. Optimum dressing system parameters

\begin{tabular}{|c|c|c|c|c|c|c|}
\hline \multirow[b]{2}{*}{$\begin{array}{l}\text { Synthetic } \\
\text { diamonds }\end{array}$} & \multirow[b]{2}{*}{ Dressing method } & \multicolumn{3}{|c|}{ Dressing conditions } & \multirow[b]{2}{*}{$\begin{array}{l}\text { Grit sizes ratio } \\
\qquad q_{g}\end{array}$} & \multirow{2}{*}{$\begin{array}{c}\text { Generalized utility } \\
\text { function } \\
\Phi_{G, i h}\end{array}$} \\
\hline & & $\begin{array}{l}\text { Radial feed rate } \\
f_{r d}[\mathrm{~mm} / \mathrm{min}]\end{array}$ & $\begin{array}{c}\text { Dressing speed ratio } \\
\qquad q_{d} \\
\end{array}$ & $\begin{array}{c}\text { Dress-out time } \\
t_{d}[\mathrm{~s}]\end{array}$ & & \\
\hline \multirow{2}{*}{ AC32 } & Uni-directional & 0.2 & 0.8 & 5.7 & 2.53 & 0.6984 \\
\hline & Counter-directional & 0.2 & 0.2 & 3.5 & 2.56 & 0.6873 \\
\hline \multirow{2}{*}{ AC80 } & Uni-directional & 1.4 & 0.8 & 9 & 2.21 & 0.7534 \\
\hline & Counter-directional & 0.2 & 0.2 & 1 & 2.56 & 0.6736 \\
\hline
\end{tabular}

Table 10. Comparison of experimental and predicted values of the grinding process response variables

\begin{tabular}{|c|c|c|c|c|c|c|c|c|c|c|c|c|c|}
\hline \multirow[b]{2}{*}{$\begin{array}{l}\text { Synthetic } \\
\text { diamonds }\end{array}$} & \multicolumn{5}{|c|}{ Optimum dressing system parameters } & \multicolumn{2}{|c|}{$\begin{array}{l}\text { Roughness } \\
\text { of the ground } \\
\text { surface } \\
R a_{w}[\mu \mathrm{m}]\end{array}$} & \multicolumn{2}{|c|}{$\begin{array}{c}\text { Accuracy of the } \\
\text { ground surface } \\
\qquad \delta_{w}[\mu \mathrm{m}]\end{array}$} & \multicolumn{2}{|c|}{$\begin{array}{c}\text { Grinding wheel } \\
\text { lifetime } \\
T_{S} \text { [min] }\end{array}$} & \multicolumn{2}{|c|}{$\begin{array}{c}\text { Net costs } \\
\text { of grinding } \\
\text { operation } \\
C[€ / p c]\end{array}$} \\
\hline & Dressing method & $\underset{[\mathrm{mm} / \mathrm{min}]}{f_{r d}}$ & $q_{d}$ & $\begin{array}{l}t_{d} \\
{[\mathrm{~s}]}\end{array}$ & $q_{g}$ & EV & PV & EV & PV & EV & PV & EV & PV \\
\hline \multirow{2}{*}{ AC32 } & Uni-directional & 0.2 & 0.8 & 5.7 & 2.53 & 0.38 & 0.369 & 6.81 & 6.507 & 39.6 & 38.05 & 0.03 & 0.0289 \\
\hline & Counter-directional & 0.2 & 0.2 & 3.5 & 2.56 & 0.3 & 0.285 & 9.35 & 9.182 & 34.7 & 33.36 & 0.024 & 0.0229 \\
\hline \multirow{2}{*}{ AC80 } & Counter-directional & 1.4 & 0.8 & 9 & 2.21 & 0.62 & 0.624 & 7.2 & 7.535 & 44.0 & 45.15 & 0.027 & 0.0271 \\
\hline & Uni-directional & 0.2 & 0.2 & 1 & 2.56 & 0.61 & 0.588 & 8.42 & 8.524 & 52.5 & 53.46 & 0.022 & 0.0209 \\
\hline \multicolumn{14}{|c|}{ EV - experimental value; PV - predicted value } \\
\hline
\end{tabular}




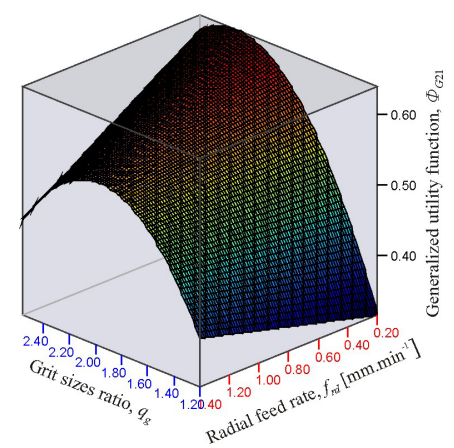

a) $q_{d}=0.5, t_{d}=5 \mathrm{~s}$

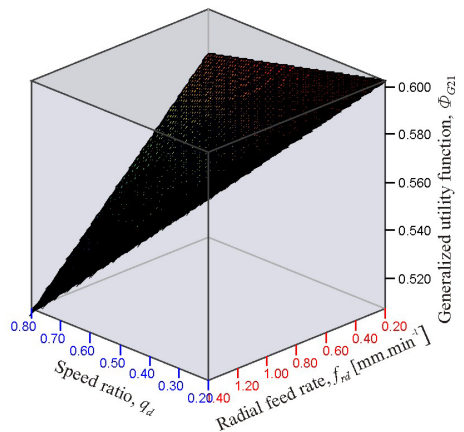

d) $q_{g}=1.88, t_{d}=5 \mathrm{~s}$

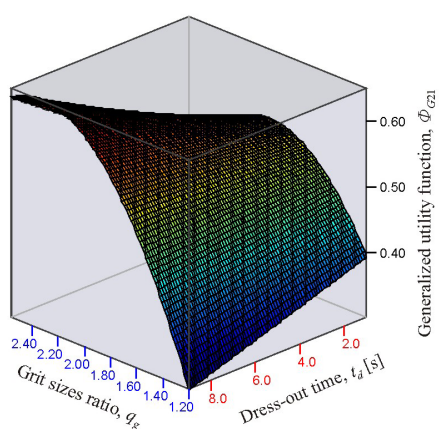

b) $q_{d}=0.5, f_{r d}=0.8 \mathrm{~mm} / \mathrm{min}$

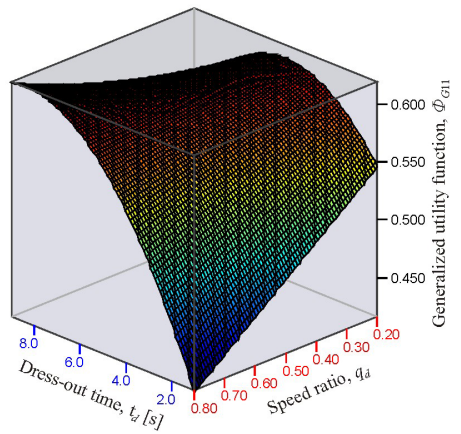

e) $q_{g}=1.88, f_{r d}=0.8 \mathrm{~mm} / \mathrm{min}$

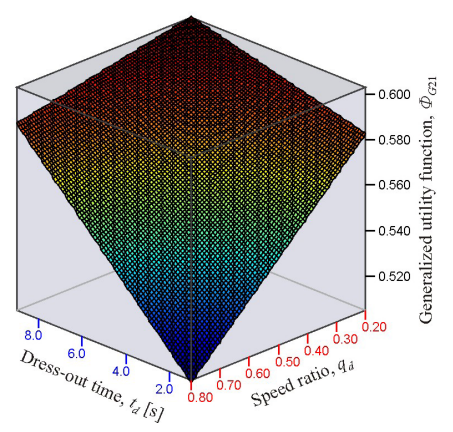

c) $q_{g}=1.88, f_{r d}=0.8 \mathrm{~mm} / \mathrm{min}$

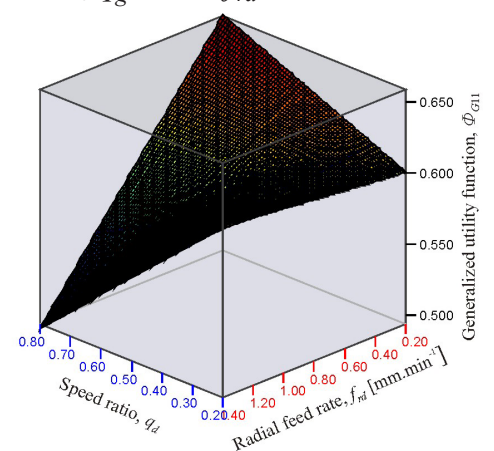

f) $q_{g}=1.88, q_{d}=0.5$

Fig. 3. Generalized utility function during uni-directional dressing with $a, b, c, d$ ) diamond roller dressers AC80, and e, f) AC32

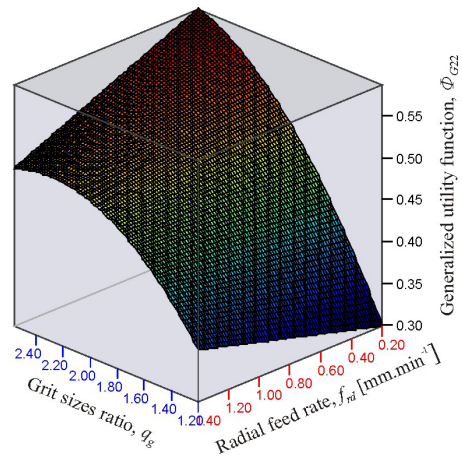

a) $q_{d}=0.5, t_{d}=5 \mathrm{~s}$

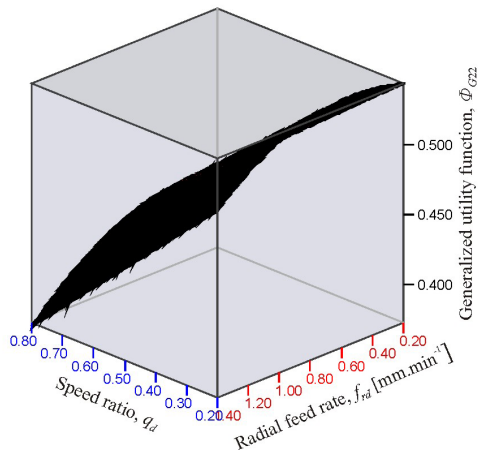

d) $q_{g}=1.88, t_{d}=5 \mathrm{~s}$

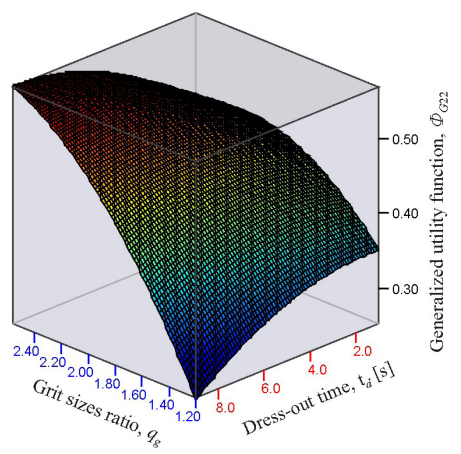

b) $q_{d}=0.5, f_{r d}=0.8 \mathrm{~mm} / \mathrm{min}$

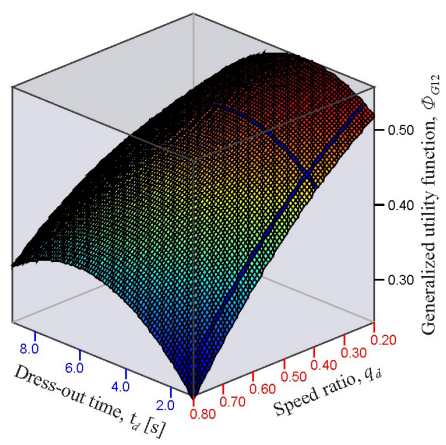

e) $q_{g}=1.88, f_{r d}=0.8 \mathrm{~mm} / \mathrm{min}$

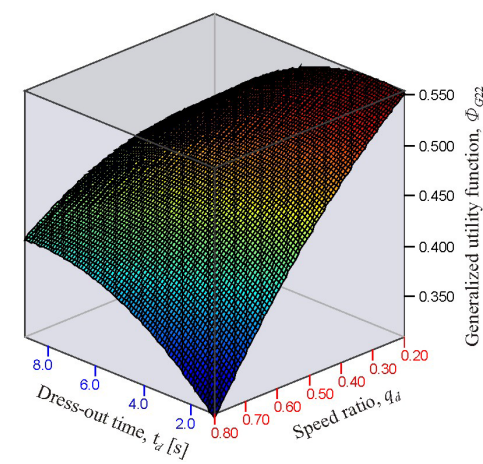

c) $q_{g}=1.88, f_{r d}=0.8 \mathrm{~mm} / \mathrm{min}$

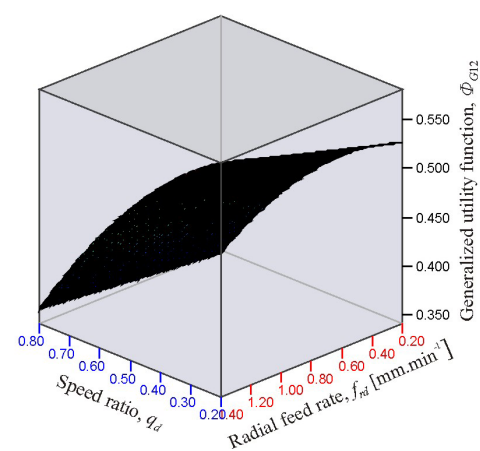

f) $q_{g}=1.88, q_{d}=0.5$

Fig. 4. Generalized utility function during counter-directional dressing with $a, b, c, d$ ) diamond roller dressers AC80, and e, f) AC32 
rate $f_{r d}$, dressing time $t_{d}$ and grit sizes ratio $q_{g}$. With regard to this, by applying genetic algorithm and employing the software product QStatLab Paretooptimum solutions to the four objective functions: $\Phi_{G 11}, \Phi_{G 12}, \Phi_{G 21}, \Phi_{G 22}$, are found, whose maximums are at different points of the studied factor space. From the found Pareto-front the following combination has been chosen as an optimum solution: $f_{r d}=0.2 \mathrm{~mm} /$ $\min , q_{d}=0.75, t_{d}=4.65 \mathrm{~s}, q_{g}=2.56$. It combines in an optimum way the largest values of the objective functions, as follows: $\Phi_{G 11}=0.6907, \Phi_{G 12}=0.5077$, $\Phi_{G 21}=6507, \quad \Phi_{G 22}=0.5073$. The determined optimum dressing system parameters provide the best combination between the roughness and accuracy of the machined surface, the grinding wheel lifetime and the manufacturing net costs of the grinding operation, as follows:

- in uni-directional dressing with diamond roller dressers of synthetic diamonds AC32: $R a_{w 11}=0.37 \mu \mathrm{m}, \delta_{w 11}=4.3 \mu \mathrm{m}, T_{s 11}=39.03 \mathrm{~min}$, $C_{11}=0.028 € / \mathrm{pc}$;

- in counter-directional dressing with diamond roller dressers of synthetic diamonds AC32: $R a_{w 12}=0.22 \mu \mathrm{m}, \delta_{w 12}=6.9 \mu \mathrm{m}, T_{s 12}=21.45 \mathrm{~min}$, $C_{12}=0.04 € / \mathrm{pc}$;

- in uni-directional dressing with diamond roller dressers of synthetic diamonds AC80: $R a_{w 21}=0.51 \mu \mathrm{m}, \delta_{w 21}=6.9 \mu \mathrm{m}, T_{s 21}=34.21 \mathrm{~min}$, $C_{21}=0.027 € / \mathrm{pc}$;

- in counter-directional dressing with diamond roller dressers of synthetic diamonds AC80: $R a_{w 22}=0.37 \mu \mathrm{m}, \delta_{w 22}=6.9 \mu \mathrm{m}, T_{s 22}=30.47 \mathrm{~min}$, $C_{22}=0.04 € / \mathrm{pc}$.

\section{CONCLUSIONS}

As a result of the conducted experimental studies, modelling and multi-objective optimization of dressing grinding wheels of aluminium oxide with diamond roller dressers of medium- and high-strength synthetic diamonds AC32 and AC80 in fine cylindrical grinding, the following results have been achieved:

(1) Adequate regression models for the response variables of the fine grinding process (roughness and accuracy of the ground surface, grinding wheel lifetime, and manufacturing net costs) depending on the dressing system parameters (radial feed rate of diamond roller dresser, dressing speed ratio, dress-out time, diamond roller dresser grit size/grinding wheel grit size ratio, type of synthetic diamonds and direction of dressing).
(2) Theoretical-experimental models have been created for determining the generalized utility function as a complex indicator characterizing the response variables of the fine grinding process. The models have been constructed for unidirectional and counter-directional dressing with diamond roller dressers of synthetic diamonds $\mathrm{AC} 32$ and AC80, and they reflect the complex impact of the dressing system parameters.

(3) With the method of the generalized utility function, the optimum dressing system parameters of uni-directional and counterdirectional dressing with diamond roller dressers of synthetic diamonds AC32 and AC80 have been determined (Table 9). On the basis of the obtained results, it can be recommended to perform dressing of grinding wheels in fine cylindrical grinding under the conditions at which the maximum value of the generalized utility function is obtained, namely: unidirectional dressing with diamond roller dressers with working layer of high-strength synthetic diamonds AC80; diamond roller dresser grit size/ grinding wheel grit size ratio $q_{g}=2.21$; radial feed rate of diamond roller dresser $f_{r d}=1.4 \mathrm{~mm} /$ min; dressing speed ratio $q_{d}=0.8$; dress-out time $t_{d}=9 \mathrm{~s}$. The grinding wheels dressing under these conditions ensures: roughness of the ground surface $0.62 \mu \mathrm{m}$, accuracy of the ground surface shape $7.2 \mu \mathrm{m}$, grinding wheel lifetime $44 \mathrm{~min}$ and manufacturing net costs of the grinding operation $0.027 € /$ pc (Table 10).

(4) With the Pareto method and by applying a genetic algorithm, the optimum dressing system parameters have been determined, valid for unidirectional and counter-directional dressing with diamond roller dressers of synthetic diamonds $\mathrm{AC} 32$ and AC80, as follows: radial feed rate of diamond roller dresser $f_{r d}=0.2 \mathrm{~mm} / \mathrm{min}$; dressing speed ratio $q_{d}=0.75$; dress-out time $t_{d}=4.65 \mathrm{~s}$ and diamond roller dresser grit size/grinding wheel grit size ratio $q_{g}=2.56$. The credibility of the determined optimum parameters has been proven by an experimental study of the response variables of the fine grinding process. It has been found that they guarantee the best combination between the roughness $\left(R a_{w} \leq 0.51 \mu \mathrm{m}\right)$ and the accuracy $\left(\delta_{w} \leq 6.9 \mu \mathrm{m}\right)$ of the ground surface, the grinding wheel lifetime $\left(T_{s} \geq 21.45 \mathrm{~min}\right)$ and the manufacturing net costs of the grinding operation $\left(C_{21} \leq 0.04 € / \mathrm{pc}\right)$. The results obtained provide possibilities for control and optimization of the fine grinding process by selecting optimum 
dressing system parameters and they can be used in all machine-building companies.

\section{ACKNOWLEDGEMENTS}

This work was supported by the Bulgarian Ministry of Education and Science and the Technical University of Gabrovo under contract No 1713M.

\section{REFERENCES}

[1] Maksoud, T.M.A., Atia, M.R. (2004). Review of intelligent grinding and dressing operations. Machining Science and Technology, vol. 8, no. 2, p. 263-276, Dol:10.1081/MST200028748.

[2] Malkin, S. (2008). Grinding Technology: Theory and Application of Machining with Abrasives. Industrial Press, New York.

[3] Puerto, P., Fernández, R., Madariaga, J., Arana, J., Gallego, I. (2013). Evolution of surface roughness in grinding and its relationship with the dressing parameters and the radial wear. Procedia Engineering, vol. 63, p. 174-182, D0l:10.1016/j. proeng.2013.08.181.

[4] Ostaševičius, V., Jūrènas, V., Vilkauskas, A., Balevičius, G., Senkus, A., Jotautienè, E. (2017). A novel excitation approach to ultrasonically-assisted cylindrical grinding. Strojniški vestnik - Journal of Mechanical Engineering, vol. 63, no. 12, p. 696704, D0I:10.5545/sv-jme.2016.4196.

[5] Cebalq, R. (1988). Optimization of the creep-feed grinding process. International Journal of Production Research, vol. 26, no. 10, p. 1665-1670, D0I:10.1080/00207548808947980.

[6] Baseri, H., Rezaei, S.M., Rahimi, A., Saadat, M. (2008). Analysis of the disc dressing effects on grinding performance - part 1: Simulation of the disc dressed wheel surface. Machining Science and Technology, vol. 12, no. 2, p. 183196, DOI:10.1080/10910340802067395.

[7] Baseri, H., Rezaei, S.M., Rahimi, A., Saadat, M. (2008). Analysis of the disc dressing effects on grinding performance - part 2: Effects of the wheel topographical parameters on the specific energy and workpiece surface roughness. Machining Science and Technology, vol. 12, no. 2, p. 197-213, DOI:10.1080/10910340802067429.

[8] Baseri, H. (2012). Simulated annealing based optimization of dressing conditions for increasing the grinding performance. International Journal of Advanced Manufacturing Technology, vol. 59, no. 5-8, p. 531-538, D0l:10.1007/s00170-011-3518-9.

[9] Wegener, K, Hoffmeister, H.-W., Karpuschewski, B., Kuster, F., Hahmann, W.-C., Rabiey, M. (2011) Conditioning and monitoring of grinding wheels. CIRP Annals, vol. 60, no. 2, p. 757-777, DOI:10.1016/j.cirp.2011.05.003.

[10] Palmer, J., Ghadbeigi, H., Novovic, D., Curtis, D. (2018). An experimental study of the effects of dressing parameters on the topography of grinding wheels during roller dressing. Journal of Manufacturing Processes, vol. 31, p. 348-355, DOI:10.1016/J.jmapro.2017.11.025.

[11] Chen, Y., Chen, X., Xu, X., Yu, G. (2018). Effect of energy consumption in the contact zone on machining condition optimization in precision surface grinding. Strojniški vestnik Journal of Mechanical Engineering, vol. 64, no. 4, p. 233-244, DOI:10.5545/sv-jme.2017.4995.

[12] Jiang, J.L., Ge, P.Q., Bi, W.B., Zhang, L., Wang, D.X., Zhang, Y. (2013). 2D/3D ground surface topography modeling considering dressing and wear effects in grinding process. International Journal of Machine Tools and Manufacture, vol. 74, p. 29-40, D0I:10.1016/j.ijmachtools.2013.07.002.

[13] Saad, A., Bauer, R., Warkentin, A. (2010). Investigation of single-point dressing overlap ratio and diamond-roll dressing interference angle on surface roughness in grinding. Transactions of the Canadian Society for Mechanical Engineering, vol. 34, no. 2, p. 295-308, Dol:10.1139/ tcsme-2010-0018.

[14] Lefebvre, A., Sinot, 0., Torrance, A. (2013). Optimization of dressing conditions for a resin-bonded diamond wheel by topography analysis. Machining Science and Technology, vol. 17, no. 2, p. 312-324, DOI:10.1080/10910344.2013.781364.

[15] Zylka, L., Burek, J., Mazur, D. (2017). Diagnostic of peripheral longitudinal grinding by using acoustic emission signal. Advances in Production Engineering \& Management, vol. 12, no. 3, p. 221-232, DOl:10.14743/apem2017.3.253.

[16] Linke, B. (2008). Dressing process model for vitrified bonded grinding wheels. CIRP Annals, vol. 57, no. 1, p. 345-348, DOI:10.1016/j.cirp.2008.03.083.

[17] Lee, K.-M., Hsu, M.-R., Chou, J.-H., Guo, C.-Y. (2011). Improved differential evolution approach for optimization of surface grinding process. Expert Systems with Applications, vol. 38, no. 5, p. 5680-5686, D0l:10.1016/j.eswa.2010.10.067.

[18] Slowik, A., Slowik, J. (2008). Multi-objective optimization of surface grinding process with the use of evolutionary algorithm with remembered Pareto set. International Journal of Advanced Manufacturing Technology, vol. 37, no. 7-8, p. 657-669, DOl:10.1007/s00170-007-1013-0.

[19] Saravanan, R., Sachithanandam, M. (2001). Genetic algorithm (GA) for multivariable surface grinding process optimisation using a multi-objective function model. International Journal of Advanced Manufacturing Technology, vol. 17, no. 5, p. 330338, D0I:10.1007/s001700170167.

[20] Zhang, G., Liu, M., Li, J., Ming, W.Y., Shao, X.Y., Huang, Y. (2014). Multi-objective optimization for surface grinding process using a hybrid particle swarm optimization algorithm. International Journal of Advanced Manufacturing Technology, vol. 71, no. 9-12, p. 1861-1872, D0l:10.1007/s00170-0135571-z.

[21] Krishna, A., Rao, K. (2006). Multi-objective optimization of surface grinding operations using scatter search approach. International Journal of Advanced Manufacturing Technology, vol. 29, no. 5-6, p. 475-480, DOl:10.1007/BF02729099.

[22] Baskar, N., Saravanan, R., Asokan, P., Prabhaharan, G. (2004). Ants colony algorithm approach for multi-objective optimisation of surface grinding operations. International Journal of Advanced Manufacturing Technology, vol. 23, no. 5-6, p. 311-317, D01:10.1007/s00170-002-1533-6.

[23] Amitay, G., Malkin, S., Koren, Y. (1981). Adaptive control optimization of grinding. Journal of Engineering for Industry, vol. 103, no. 1, p. 103-108, Dol:10.1115/1.3184449. 
[24] Klancnik, S., Brezocnik, M., Balic, J. (2016). Intelligent CAD/CAM system for programming of CNC machine tools. International Journal of Simulation Modelling, vol. 15, no. 1, p. 109-120, D0I:10.2507/IJSIMM15(1)9.330.

[25] Aleksandrova, I. (2016). Optimization of the dressing parameters in cylindrical grinding based on a generalized utility function. Chinese Journal of Mechanical Engineering, vol. 29, no. 1, p. 63-73, D0l:10.3901/CJME.2015.1103.130.

[26] Catalogue of the Abrasive Tools Factory - Berkovitsa (2018). from https://0501.nccdn.net/4_2/000/000/084/3b1/ZAl2018.pdf, accessed on 2018-10-30.

[27] GOST 9206-80 (1980). Diamond powders. Technical Conditions. USSR State Committee for Standard. Moscow. (in Russian)

[28] DSTU $3292-95$ (1995). Synthetic Diamond Powders. General Technical Conditions. State Standard of Ucraine, Kiev. (in Russian)

[29] Industrial Diamond Association of America, Inc. A Review of Diamond Sizing and Standards, from http:// www.nationalresearchcompany.com/wp-content/ uploads/2015/05/Diamond_Sizing_and_Standards.pdf, accessed on 2018-12-07.

[30] Aleksandrova, I. (1995). Optimization of Dressing Process of Abrasive Tools with Diamond Profiling Rollers. PhD thesis, Technical University of Gabrovo, Gabrovo. (in Bulgarian)

[31] Aleksandrova, I. (2010). Methodology of modeling, identification and control of grinding process. Inzhenerni nauki, no. 1, p. 60-72. (in Bulgarian)

[32] Aleksandrov, A., Aleksandrova, I. (2012). Theory of Experiment. Express, Gabrovo. (in Bulgarian)

[33] Schmitt, R. (1968) Abrichten von Schleifscheiben mit diamantbestückten Rollen. PhD thesis, TU Braunschweig. (in German)

[34] Daneshi, A., Jandaghi, N., Tawakoli, T. (2014). Effect of dressing on internal cylindrical grinding. Procedia CIRP, vol. 14, p. 37-41, D0I:10.1016/j.procir.2014.03.064.

[35] Klocke, F., Linke, B. (2008). Mechanisms in the generation of grinding wheel topography by dressing. Production Engineering, vol. 2, no 2, p. 157-163, Dol:10.1007/s11740. 008-0101-9.

[36] Klocke, F., Thiermann, J., Mattfeld, P. (2015). Influence of the dressing process on grinding wheel wear. Production
Engineering, vol. 9, no 5-6, p. 563-568, Dol:10.1007/s11740015-0606-y.

[37] Vishnupad, P., Shin, Y.C. (1998). Intelligent optimization of grinding processes using fuzzy logic. Proceedings of the Institution of Mechanical Engineers, Part B: Journal of Engineering Manufacture, vol. 212, no. 8, p. 647-660, DOI:10.1243/0954405981515914.

[38] Mukherjee, I., Ray, P.K. (2006). A review of optimization techniques in metal cutting processes. Computers \& Industrial Engineering, vol. 50, no. 1-2, p. 15-34, D0l:10.1016/j. cie.2005.10.001.

[39] Yusup, N., Zain, A.M., Hashim, S.Z.M. (2012). Evolutionary techniques in optimizing machining parameters: Review and recent applications (2007-2011). Expert Systems with Applications, vol. 39, no. 10, p. 9909-9927, D0l:10.1016/j. eswa.2012.02.109.

[40] Stoyanov, S. (1993). Optimization of Manufacturing Processes. Tehnika, Sofia. (in Bulgarian)

[41] Mukherjee, I., Ray, P.K. (2008). Optimal process design of two-stage multiple responses grinding processes using desirability functions and metaheuristic technique. Applied Soft Computing, vol. 8, no. 1, p. 402-421, D0l:10.1016/j. asoc.2007.02.005.

[42] Dzebo, S., Morehouse, J.B., Melkote, S.N. (2012). A methodology for economic optimization of process parameters in centerless grinding. Machining Science and Technology, vol. 16, no. 3, p. 355-379, DOl:10.1080/10910344.2012.698958.

[43] Salmasnia, A., Kazemzadeh, R.B., Niaki, S.T.A. (2012). An approach to optimize correlated multiple responses using principal component analysis and desirability function. International Journal of Advanced Manufacturing Technology, vol. 62 , no. 5-8, p. 835-846, D0l:10.1007/s00170-011-38242.

[44] Raissi, S., Farsani, R. (2009). Statistical process optimization through multi-response surface methodology. International Scholarly and Scientific Research \& Innovation, vol. 3, no. 3, p. $247-251$.

[45] Vuchkov, I.N., Vuchkov, I.I. (2009). Statistical Methods of Quality Control, Robust Engineering, Planning, Modeling and Optimization, QStatLab, v 5.3. (in Bulgarian)

[46] Goldberg, D. (1989). Genetic Algorithms in Search, Optimization and Machine Learning. Addison Wesley Longman, London. 